\title{
Deep Radio Continuum Imaging of the Dwarf Irregular Galaxy IC 10: Tracing Star Formation and Magnetic Fields
}

\author{
V. Heesen ${ }^{1}$, U. Rau ${ }^{2}$, M. P. Rupen ${ }^{2}$, E. Brinks ${ }^{1}$, and D. A. Hunter ${ }^{3}$
}

\begin{abstract}
We exploit the vastly increased sensitivity of the Expanded Very Large Array (EVLA) to study the radio continuum and polarization properties of the poststarburst, dwarf irregular galaxy IC 10 at $6 \mathrm{~cm}$, at a linear resolution of $\sim 50 \mathrm{pc}$. We find close agreement between radio continuum and $\mathrm{H} \alpha$ emission, from the brightest H II regions to the weaker emission in the disk. A quantitative analysis shows a strictly linear correlation, where the thermal component contributes $50 \%$ to the total radio emission, the remainder being due to a non-thermal component with a surprisingly steep radio spectral index of between -0.7 and -1.0 suggesting substantial radiation losses of the cosmic-ray electrons. We confirm and clearly resolve polarized emission at the $10-20 \%$ level associated with a non-thermal superbubble, where the ordered magnetic field is possibly enhanced due to the compression of the expanding bubble. A fraction of the cosmic-ray electrons has likely escaped because the measured radio emission is a factor of 3 lower than what is suggested by the $\mathrm{H} \alpha$ inferred SFR.
\end{abstract}

Subject headings: galaxies: dwarf — galaxies: individual(IC 10) - galaxies: magnetic fields — radio continuum: galaxies

\section{Introduction}

Dwarf galaxies are the most abundant type of galaxy in the local Universe (Marzke \& da Costa 1997) and are the closest analogues to the building blocks for galaxy formation at high redshift in $\Lambda$ CDM models (see, for example, Lagos et al. 2009). Furthermore, in the "downsizing" paradigm (Hu et al. 1995; Homeier et al. 2006) dwarf galaxies increasingly dominate

\footnotetext{
${ }^{1}$ Centre for Astrophysics Research, University of Hertfordshire, Hatfield AL10 9AB, United Kingdom

${ }^{2}$ NRAO, P.V.D. Science Operations Center, National Radio Astronomy Observatory, 1003 Lopezville Road, Socorro, NM 87801, USA

${ }^{3}$ Lowell Observatory, 1400 West Mars Hill Road, Flagstaff, AZ 86001, USA
} 
the star-forming universe over time (Sawicki \& Thompson 2006), with star formation (SF) perhaps being particularly enhanced within dwarfs over the past 2 Gyr (Orban et al. 2008). Stellar winds and supernova explosions of the most massive stars can easily lead to outflows due to their weak gravitational potential. This implies that not only does the metallicity of the interstellar medium increase with cosmic time, but additionally the intergalactic medium becomes enriched at an early stage (Oppenheimer \& Davé 2006). Moreover, dwarf galaxies are supposed to have played a fundamental role in amplifying magnetic fields in the early Universe through outflowing plasma escaping from star-forming regions (Chyży et al. 2011).

Cosmic rays play an important role in galactic outflows, as they consist of relativistic particles that do not quickly lose their energy, like the hot gas. They spiral along magnetic field lines emitting highly linearly polarized, non-thermal (synchrotron) emission providing a useful tool to study magnetic field structure. This magnetic field influences how cosmic rays propagate. For example, nearby edge-on galaxies with radio haloes show a characteristic "pinched at the waist" structure in polarised emission (and sometimes even in total intensity) which is a consequence of an X-shaped magnetic field (e.g., Tüllmann et al. 2000; Heesen et al. 2009; Soida et al. 2011).

Resolved radio continuum studies of dwarf galaxies have been lacking thus far, because the objects are intrinsically faint. Chyży et al. (2003) took advantage of the excellent sensitivity of the 100-m Effelsberg telescope to study IC 10 in the radio continuum, both in total intensity and polarimetry. They found extended, diffuse emission coinciding with $\mathrm{H} \alpha$ emission, and polarized emission close to the non-thermal bubble described by Yang \& Skillman (1993). However, they were limited by their coarse resolution $(1.1)$ that prevents any detailed comparison with ancillary data. A higher resolution preliminary VLA map at $6 \mathrm{~cm}$ was published by Chyży (2005). Chyży et al. (2000), using the VLA, found in NGC 4449 despite its weak rotation a surprisingly regular magnetic field showing hints of spiral structure and "fans" possibly connected to outflows. Kepley et al. (2010), again using the VLA, studied the starburst dwarf NGC 1569 which has a strong radial field, likely shaped by the outflowing gas.

With the new capabilities of the expanded very large array (EVLA) and much improved sensitivity we can study the weak extended emission in dwarf galaxies. In this letter we present first results from observations of IC 10 with the EVLA at C-band, using the full $2 \mathrm{GHz}$ of bandwidth that was available at the time of observation. Additional observations at $\mathrm{L}^{-}, \mathrm{X}-$, and $\mathrm{Ka}-$ band, along with a full interpretation of the data will be published in a forthcoming paper.

IC 10 is a perfect candidate for an in depth study of a dwarf galaxy at radio continuum wavelengths because it is relatively bright and nearby, being a member of the Local Group 
(Sanna et al. 2008). It has a high star formation rate and a high density of Wolf-Rayet stars and is regarded to be in a starburst phase (Massey \& Holmes 2002; Crowther et al. 2003). The aftermath of massive star formation leaves its imprint on the ISM in the form of expanding bubbles and shells that can be traced in H I and H $\alpha$. Wilcots \& Miller (1998) found holes in $\mathrm{H}$ I and $\mathrm{H} \alpha$ emission with large filaments in $\mathrm{H} \alpha$ extending up to a few hundred parsec away from the main body (Hunter et al. 2006), suggesting outflows. The H I emission is asymmetric with respect to the major axis. A feature visible in velocity maps suggests ongoing accretion of neutral hydrogen in the outer parts of the galaxy. There is a detection of diffuse hot $\mathrm{X}$-ray emitting gas in this galaxy that is aligned with the main stellar bar (Wang et al. 2005). Yang \& Skillman (1993) found a region with high radio continuum brightness and identified it as a non-thermal bubble prior to the break out. Lozinskaya \& Moiseev (2007) speculated that it could be generated by a hypernova rather than by a collection of supernovae.

As IC 10 is close to the Galactic midplane $\left(b=-3^{\circ} .3\right)$, estimates of its distance are uncertain due to the unknown level of absorption. Here, we use a conservative distance of $1 \mathrm{Mpc}$ in line with the most recent results that indicate a likely distance at best slightly less than 1 Mpc (Kim et al. 2009; Sanna et al. 2008).

\section{Observations}

We observed IC 10 with the NRAO1 EVLA under the resident shared risk program (RSRO; project ID: AH1006). Our observations were taken in D-configuration on August 16, 2010. We used 2 IFs each consisting of 8 sub-bands with a bandwidth of $128 \mathrm{MHz}$ each. The sub-bands contain 64 channels at $2 \mathrm{MHz}$ resolution resulting in a total bandwidth of $2 \mathrm{GHz}$. We placed our IFs between 4.5-5.4 GHz and 6.9-7.8 GHz, respectively. We observed for $4 \mathrm{~h}$ to ensure good $(u, v)$-coverage and sensitivity to extended emission. Every $15 \mathrm{~min}$ we observed a phase calibrator (J0102+5824); we used 3C48 as our flux density (primary) calibrator, adopting the Baars et al. (1977) flux scale. In order to measure the polarization leakage (D-terms) we observed the unpolarized source 3C84. The polarization angle was calibrated using $3 \mathrm{C} 48$.

We calibrated the data with the Common Astronomy Software Applications package (CASA) as outlined in the continuum tutorial of 3C391 on the CASA homepage 2 . In all

\footnotetext{
${ }^{1}$ The National Radio Astronomy Observatory is a facility of the National Science Foundation operated under cooperative agreement by Associated Universities, Inc.

${ }^{2}$ http://casaguides.nrao.edu/index.php?title=EVLA_Continuum_Tutorial_3C391
} 
subbands, leakages of $5 \%-15 \%$ before calibration were reduced to $0.2 \%$ (variation across channels) after calibration. R-L polarization angles varied by $5^{\circ}-10^{\circ}$ with a constant slope across channels before calibration, and went down to $1^{\circ}-2^{\circ}$ variations across channels after calibration. We constructed Stokes $I, Q$, and $U$ images using the Multi-Scale Clean algorithm (Cornwell 2008; Rich et al. 2008) in CASA that models extended emission accurately, but assumes a flat sky-spectrum. In Stokes $I$ the integrated flux density was $131 \mathrm{mJy}$, with a peak brightness of $11.3 \mathrm{mJy}$. The integrated polarized intensity is $3.0 \mathrm{mJy}$ with peak brightness of $-58 \mu \mathrm{Jy}$ and $47 \mu \mathrm{Jy}$ in Stokes $Q$ and $U$, respectively. The target rms noise calculated for this observation with $\sim 3$ hours on-source was $4 \mu \mathrm{Jy}$. The rms noise in the Stokes $Q$ and $U$ images was $5 \mu \mathrm{Jy}$, but the Stokes $I$ image was affected by deconvolution errors at the $40 \mu \mathrm{Jy}$ level. These errors were at the expected level for continuum imaging when ignoring spectral structure (average dynamic range $~ 300$ for a spectral index of -0.7 across a $50 \%$ bandwidth, $4.5-7.8 \mathrm{GHz})$.

Stokes I imaging was repeated with the Multi-Scale Multi-Frequency Synthesis (MSMFS) algorithm (Rau 2010; Rau \& Cornwell 2011) which simultaneously solves for spatial and spectral structure during wide-band image reconstruction, and reached an rms noise of $15 \mu \mathrm{Jy}$. This wide-band model was then used to perform one round of amplitude and phase self-calibration, and the resulting image (shown in greyscale in Fig.11) achieved the rms noise of $5 \mu \mathrm{Jy}$ only $20 \%$ higher than the theoretically expected level. We note that the rms is identical for Stokes $I, Q$, and $U$, so that we are not dominated by the signal-to-noise ratio in Stokes $I$; the self-calibration is sufficiently accurate.

A radio spectral index image was produced by MS-MFS as well, and a post-deconvolution wide-band primary beam correction was applied to remove the effect of the frequencydependent primary beam. One of the goals of this RSRO project was to test the accuracy of this approach. Therefore, this spectral index map was compared with a low-resolution spectral index map constructed within AIPS from primary-beam corrected Stokes $I$ images at each sub-band and smoothed to the resolution of the lowest sub-band. The results agreed, confirming that for this source, wide-band imaging via MS-MFS followed by a postdeconvolution primary beam correction was able to recover the same spectral information as traditional methods, but at a much improved angular resolution.

The Stokes $Q$ and $U$ images were taken into AIPS3, and maps of the polarized intensity were constructed using comb with option polc that applies a correction to the noise bias. We also created the maps of the polarization angle in comb using the option pola. We rotate the polarization $\left(\mathrm{E}-\right.$ ) vectors by $90^{\circ}$ to show the orientation of the magnetic field, where we

\footnotetext{
${ }^{3}$ AIPS, the Astronomical Image Processing Software, is free software from NRAO.
} 
neglect any contribution of Faraday rotation. We tested whether the structure of Stokes $Q$ and $U$ changed with frequency by making images for the 16 individual sub-bands and found the change to be small, indicating negligible Faraday rotation within our frequency range.

We calculated the degree of polarization and found values between $5-20 \%$. These values are at least a factor of 5 larger than what we would expect from instrumental polarization which is around $1 \%$ for the VLA beam (Condon et al. 1998). This was further checked by us as we carried out separate test observations with 3C84 at the half-power point in C-band and found values there of at most 3\%. However, because of beam smearing and the fact that our source lies well within the half-power point, we expect instrumental polarization to be considerably smaller. We therefore conclude that our measured polarization is dominated by the intrinsic polarization of our source.

\section{Results}

Figure1 shows our total power continuum radio map at $6 \mathrm{~cm}$ with a resolution of $9 . .4 \times$ $7^{\prime \prime}$.3. Contours start at $3 \times$ the rms noise level and increase by a factor of about 2 with every further contour. We choose a logarithmic grey scale to show the noise floor that is evenly distributed over the full extent of the map. To our knowledge this is the deepest radio continuum map of a dwarf galaxy yet made, going deeper than the preliminary VLA map, also at $6 \mathrm{~cm}$, published by Chyży (2005) which reaches a noise of $9 \mu \mathrm{Jy}$ (Chyży, priv. comm.). The deep VLA map of NGC 1569 at $6 \mathrm{~cm}$ by Kepley et al. (2010) has an rms noise of $8.5 \mu \mathrm{Jy}_{\text {beam }}{ }^{-1}$ but at a smaller beam size of $\sim 4^{\prime \prime}$ and is much less sensitive to extended emission. Although there are no artefacts visible in the figure that could be ascribed to missing short spacings, it can not be ruled out that we miss low-level, extended emission. We find a total flux of 131 mJy whereas we expect, based on interpolating single dish observations at $10.45 \mathrm{GHz}$ and $2.64 \mathrm{GHz}$ (Chyży et al. 2003, 2011) $190 \mathrm{mJy}$. We could thus be missing up to $30 \%$ in total power.

We compare in Fig. 2 the radio continuum emission with a deep H $\alpha$ map of Hunter \& Elmegreen (2004). The $\mathrm{H} \alpha$ is shown as a logarithmic grey-scale map to enhance weak emission features away from the main stellar body. The radio and $\mathrm{H} \alpha$ emission follow each other closely. The radio continuum peaks at the location of the $\mathrm{H} \alpha$ emission maxima that trace $\mathrm{H}$ II complexes. Moreover, thanks to the exquisite dynamic range, we can compare the radio emission with extended $\mathrm{H} \alpha$ emission, including various shells and filaments. The large filament in the West with a length of $600 \mathrm{pc}$ is the most prominent feature but we are sensitive enough to also detect the much weaker filaments in the Eastern half. The most prominent hole in the radio continuum is found at $\mathrm{RA}=00^{\mathrm{h}} 20^{\mathrm{m}} 23^{\mathrm{s}}, \mathrm{DEC}=59^{\circ} 18^{\prime} .4$ and is fully contained, both in $\mathrm{H} \alpha$ 
and radio. It was identified by Wilcots \& Miller (1998) to be the most prominent hole in H I emission with hints of expansion.

The close correlation between the radio continuum and $\mathrm{H} \alpha$ emission suggests a large fraction of thermal radio emission from free-free emission. This is borne out by the radio spectral index map presented in Fig. 3. The thermal emission has a radio spectral index of $\alpha=-0.1\left(S \propto \nu^{\alpha}\right)$, whereas the non-thermal synchrotron emission has a spectral index of -0.7 (provided the cosmic ray electrons are young, otherwise the spectrum is yet steeper). We find that almost all compact resolved sources within the galaxy have a flat spectral index between -0.1 and -0.2 indicative of a dominant thermal component. These are the compact H II regions with intense star formation visible in $\mathrm{H} \alpha$ emission. We note that our radio spectral index map obtained via MS-MFS has a better resolution than that obtained with the conventional method, where the resolution is limited by the low frequency map. This allows us to reliably identify the compact sources in the radio spectral index map that otherwise would be confused in the lower resolution version.

Away from the H II regions the radio spectral index steepens to values between -0.7 and -1 . This can only be explained with a non-thermal synchrotron component. Also, this requires synchrotron and inverse Compton losses to be important for the cosmic-ray electrons as the radio spectral index is significantly steeper than -0.7 , the value expected for young cosmic-ray electrons that have been accelerated in supernova shock waves. This result is surprising as cosmic rays in starburst dwarf galaxies are supposed to be advected in the outflow and their residence time would be short compared to their cooling time. The radio spectral index steepens even more dramatically at the south-eastern edge (RA $=00^{\mathrm{h}} 20^{\mathrm{m}} 30^{\mathrm{s}}$, DEC $\left.=59^{\circ} 16^{\prime} \cdot 5\right)$ where the superbubble of Yang \& Skillman (1993) resides. There the values are between -1 and -1.2 indicating dominating synchrotron and inverse Compton losses.

The map of the structure of the regular magnetic field is presented in Fig.4. We show the orientation of the large-scale magnetic field as measured from the linear polarization, where the length of the vectors is proportional to the polarized intensity. The degree of polarization in this region is $10-20 \%$ indicating a significant ordered magnetic field component possibly enhanced by compression by the expanding superbubble. The magnetic field orientation is approximately NE-SW, where the polarized emission extends to the southern tip of the galaxy.

We also detect a magnetic field at the location of the two brightest $H$ II regions in the southeastern part. At this position, away from the superbubble, the magnetic field orientation is more aligned with the stellar body. We can not decide, based on the current data, if the magnetic field traverses the H II regions, because the field could also lie in front 
of or behind them, in a thick gas layer as is expected for dwarf galaxies. At this resolution we do not detect a magnetic field that extends the full extent of the galaxy. We note that the location of our polarized intensity and the orientation of the magnetic field agrees well with that measured with the 100-m Effelsberg telescope (Chyży et al. 2003) and the map of Chyży (2005), lending confidence to the performance of the EVLA and the CASA data reduction package.

\section{Discussion}

We compared the flux densities of the radio emission and $\mathrm{H} \alpha$ emission averaged in $50 \mathrm{pc}\left(10^{\prime \prime}\right)$ boxes. The radio and $\mathrm{H} \alpha$ maps were convolved with a Gaussian to a HPBW of $50 \mathrm{pc}$ prior to averaging. The result is presented in Fig. 5. The $\mathrm{H} \alpha$ map was corrected for foreground absorption using $\mathrm{E}(\mathrm{B}-\mathrm{V})=0.75$ (Burstein \& Heiles 1984). We clipped the radio data below $5 \times$ the $\mathrm{rms}$ noise level, to ensure we only use reliable data points and to moderate the influence of missing flux density on our interferometric observations. A power law fit in the double logarithmic plot results in a power law index of $0.97 \pm 0.03$ and is therefore consistent with a strictly linear relation between the two variables. The dashed line shows the relation if the radio flux density were purely thermal free-free emission calculated from the $\mathrm{H} \alpha$ flux density (e.g. Deeg et al. 1997, Eq. 3), assuming an electron temperature of $10^{4} \mathrm{~K}$. The thermal flux density alone is a factor 2 lower than the measured radio flux density. This is consistent with a thermal fraction of $50 \%$ in this galaxy as indicated by the flat radio spectral index.

The linear relation between the radio and the $\mathrm{H} \alpha$ brightness means that we can use both equally well as star formation tracers. The star formation rate (SFR) from the radio continuum is given by Condon (1992) and can be converted to a star formation rate density. If we convert the $\mathrm{H} \alpha$ emission into a star formation rate by the relation given in Kennicutt (1998), we can compare the two measurements. The radio star formation rate is a factor of 3 lower than that implied by $\mathrm{H} \alpha$. This means that IC 10 lies beneath the radio-SFR correlation and is radio dim. Further studies are required to investigate how this corresponds to the thermal and non-thermal fraction of the radio emission. In theory we expect that the high frequency observations are a better tracer for the thermal component, especially since they require no correction for internal or foreground extinction. One goal of this project is to establish how well we can use radio emission as star formation tracer even in such extreme cases as starburst dwarf galaxies.

Leroy et al. (2005) found that the molecular depletion time is short in IC 10 and that the inferred SFRs are too high for the molecular gas content. This may be explained by a 
starburst although it is not clear how much gas is hidden and not traced by the CO sub-mm rotational lines (Madden et al. 1997; Bolatto et al. 2000). As in many dwarf galaxies the knowledge of the molecular gas content is still sparse, the development of an alternative SFR tracer becomes even more important than for normal spiral galaxies.

The magnetic field structure in this galaxy lacks a strong large-scale component that would be apparent in radio continuum polarization. This fits the expectation that the standard $\alpha \Omega$-dynamo needs shear to operate as near-solid body rotation will provide only weak amplification (e.g. Widrow 2002). Such conditions favour studies of local magnetic field amplification mechanisms that are normally difficult to observe in spiral galaxies, as in these systems shear-enhanced disk magnetic fields dominate. The non-thermal superbubble in IC 10 offers the possibility to study what role cosmic rays and magnetic fields play in the disk-halo interface in porous interstellar medium conditions. State of the art MHD

simulations by de Avillez \& Breitschwerdt (2005) suggest that the magnetic field gets swept up in the walls of the shells but can not prevent a superbubble break-out. Eventually the full set of observations will allow us to get a handle on the energetics of the superbubble and put these models to the test.

V.H. is funded by the Science and Technology Facilities Council (STFC) via a rolling grant to the Centre for Astrophysics Research. V.H. gratefully acknowledges the hospitality of NRAO, as provided under the RSRO scheme. D.A.H. is grateful for funding provided by the National Science Foundation through grant AST-0707563.

\section{REFERENCES}

Baars, J. W. M., Genzel, R., Pauliny-Toth, I. I. K., \& Witzel, A. 1977, A\&A, 61, 99

Bolatto, A. D., Jackson, J. M., Wilson, C. D., \& Moriarty-Schieven, G. 2000, ApJ, 532, 909

Brentjens, M. A., \& de Bruyn, A. G. 2005, A\&A, 441, 1217

Burstein, D., \& Heiles, C. 1984, ApJS, 54, 33

Chyży, K. T. 2005, Starbursts: From 30 Doradus to Lyman Break Galaxies, 329, 12P

Chyży, K. T., Beck, R., Kohle, S., Klein, U., \& Urbanik, M. 2000, A\&A, 355, 128

Chyży, K. T., Knapik, J., Bomans, D. J., Klein, U., Beck, R., Soida, M., \& Urbanik, M. 2003, A\&A, 405, 513 
Chyży, K. T., Weżgowiec, M., Beck, R., \& Bomans, D. J. 2011, A\&A, 529, A94

Condon, J. J. 1992, ARA\&A, 30, 575

Condon, J. J., Cotton, W. D., Greisen, E. W., Yin, Q. F., Perley, R. A., Taylor, G. B., \& Broderick, J. J. 1998, AJ, 115, 1693

Cornwell, T. J. 2008, IEEE Journal of Selected Topics in Signal Processing, Vol. 2, Issue 5, 793

Crowther, P. A., Drissen, L., Abbott, J. B., Royer, P., \& Smartt, S. J. 2003, A\&A, 404, 483

Deeg, H.--J., Duric, N., \& Brinks, E. 1997, A\&A, 323, 323

de Avillez, M. A. \& Breitschwerdt, D. 2005, A\&A, 436, 586

Heesen, V., Krause, M., Beck, R., \& Dettmar, R.-J. 2009, 506, 1123

Homeier, N. L., et al. 2006, ApJ, 647, 256

Hu, E. M., Kim, T.-S., Cowie, L. L., Songaila, A., \& Rauch, M. 1995, AJ, 110, 1526

Hunter, D. A., Elmegreen, B. G., \& Martin, E. 2006, AJ, 132, 801

Hunter, D. A., \& Elmegreen, B. G. 2004, AJ, 128, 2170

Kennicutt, Jr., R. C. 1998, ARA\&A, 36, 189

Kepley, A. A., Mühle, S., Everett, J., Zweibel, E. G., Wilcots, E. M., \& Klein, U. 2010, ApJ, 712,536

Kim, M., Kim, E., Hwang, N., Lee, M. G., Im, M., Karoji, H., Noumaru, J., \& Tanaka, I. 2009, ApJ, 703, 816

Lagos, C. D. P., Padilla, N. D., \& Cora, S. A. 2009, MNRAS, 397, L31

Leroy, A., Bolatto, A., Walter, F., \& Blitz, L. 2006, ApJ, 643, 825

Lozinskaya, T. A., \& Moiseev, A. V. 2007, MNRAS, 381, L26

Madden, S. C., Poglitsch, A., Geis, N., Stacey, G. J., \& Townes, C. H. 1997, ApJ, 483, 200

Marzke, R. O., \& da Costa, L. N. 1997, AJ, 113, 185

Massey, P., \& Holmes, S. 2002, ApJ, 580, L35 
Oppenheimer, B. D., \& Davé, R. 2006, MNRAS, 373, 1265

Orban, C., Gnedin, O. Y., Weisz, D. R., Skillman, E. D., Dolphin, A. E., \& Holtzman, J. A. 2008, ApJ, 686, 1030

Rau, U. 2010, PhD Dissertation, New Mexico Institute of Mining and Technology, USA

Rau, U., \& Cornwell, T. J. 2011, accepted by A\&A, arXiv:1106.2745

Rich, J. W., de Blok, W. J. G., Cornwell, T. J., Brinks, E., Walter, F., Bagetakos, I., \& Kennicutt, R. C. 2008, AJ, 136, 2897

Sanna, N., Bono, G., Stetson, P. B., Monelli, M., Pietrinferni, A., Drozdovsky, I., Caputo, F., Cassisi, S., Gennaro, M., Prada Moroni, P. G., Buonanno, R., Corsi, C. E., Degl'Innocenti, S., Ferraro, I., Iannicola, G., Nonino, M., Pulone, L., Romaniello, M., \& Walker, A. R. 2008, ApJ, 688, L69

Sawicki, M., \& Thompson, D. 2006, ApJ, 648, 299

Soida, M., Krause, M. Dettmar, R.-J., \& Urbanik, M. 2011, accepted by A\&A

Thurow, J. C., \& Wilcots, E. M. 2005, ApJ, 129, 745

Tüllmann, R., Dettmar, R.--J., Soida, M. Urbanik, M., \& Rossa, J. 2000, ApJ, 364, 36

Wang, Q. D., \& Whitaker, K. E., Williams, R. 2005, MNRAS, 362, 1065

Widrow, L. M. 2002, Reviews of Modern Physics, 74, 775

Wilcots, E. M., \& Miller, B. W. 1998, AJ, 116, 2363

Yang, H., \& Skillman, E. D. 1993, AJ, 106, 1448 


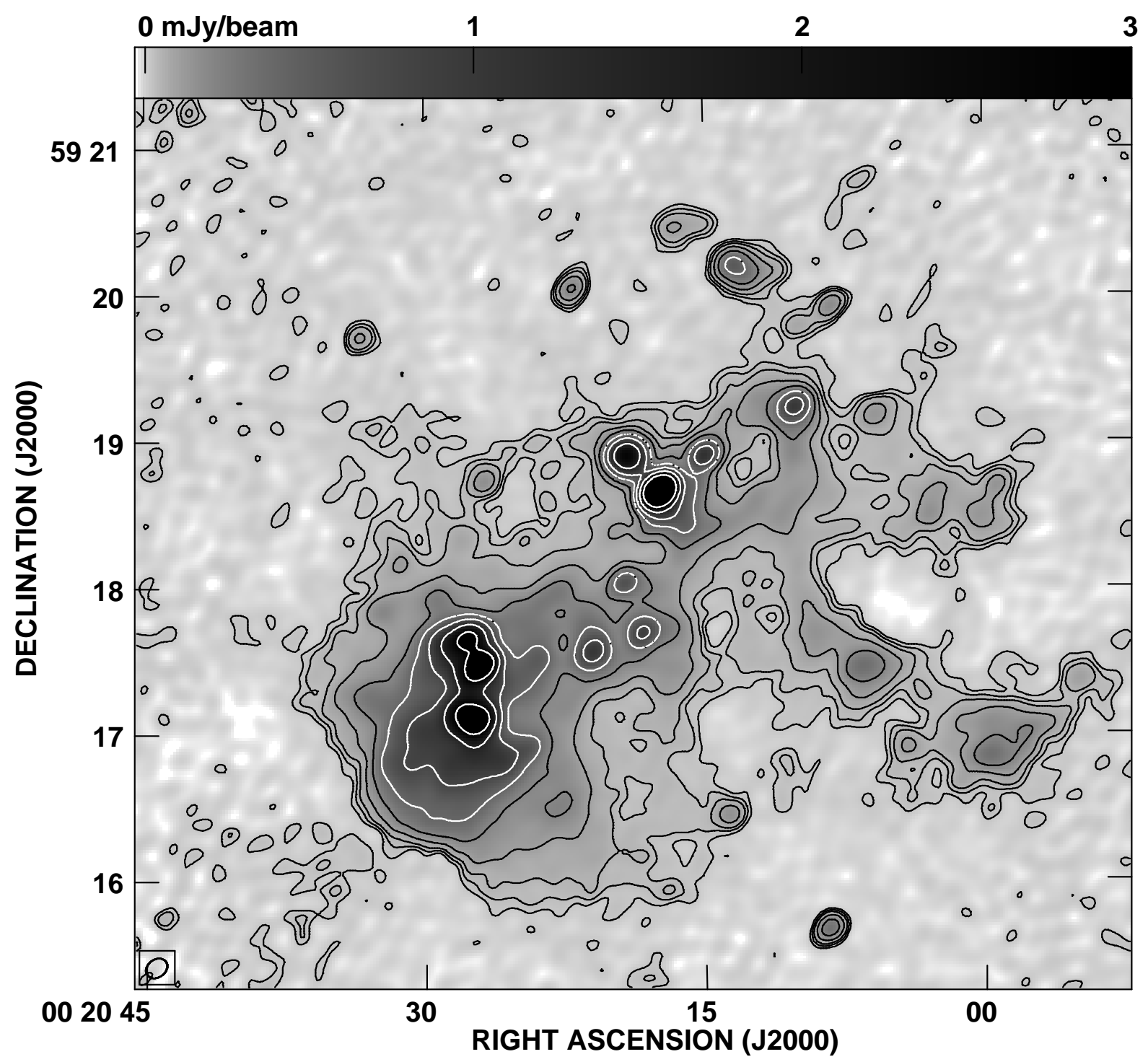

Fig. 1. - Total power radio continuum at $6 \mathrm{~cm}$ at $99^{\prime \prime} 4 \times 7^{\prime \prime} \cdot 3$ resolution as a quasi-logarithmic grey-scale image. Contours are at 3, 6, 10, 20, 40, 80, 150,300, and $600 \times 5 \mu \mathrm{Jy}_{\text {beam }}{ }^{-1}$. The rms noise level is at $5 \mu \mathrm{Jy}_{\text {beam }}{ }^{-1}$. The total power map is corrected for primary beam attenuation. 


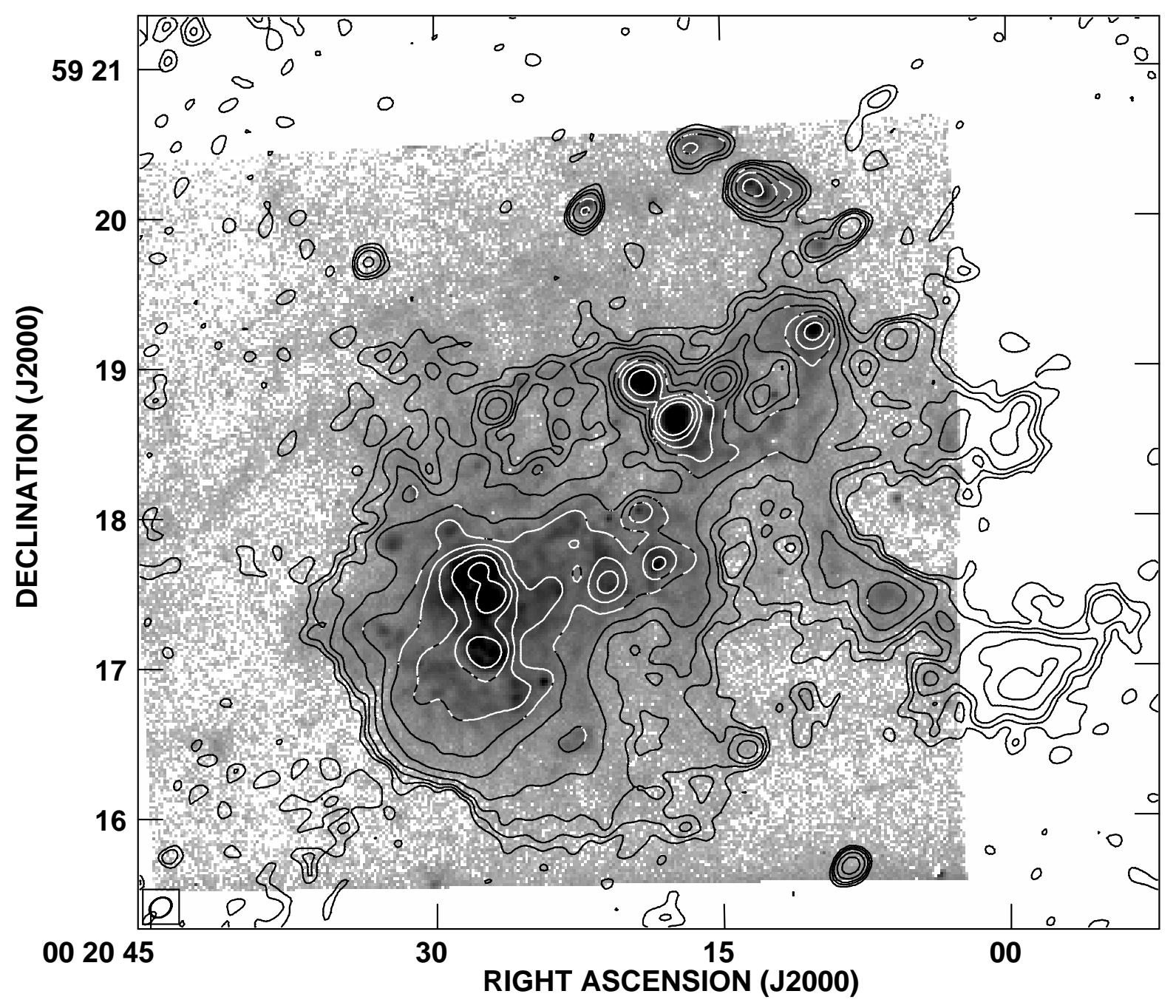

Fig. 2.- Same as Fig. 1 but as an overlay on $\mathrm{H} \alpha$ shown as a quasi-logarithmic grey-scale image. 


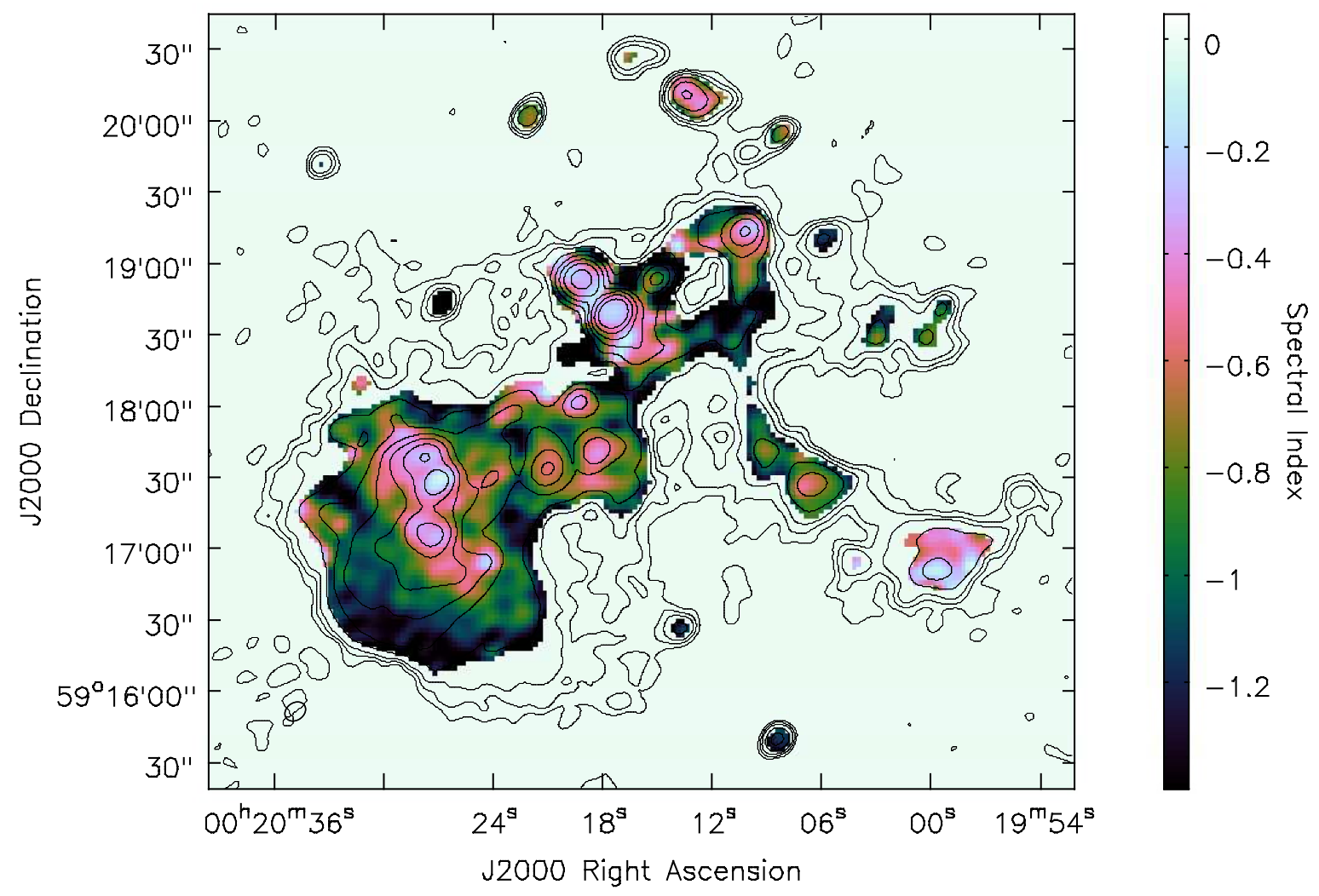

Fig. 3.- Radio spectral index map shown as a color-coded image at 9'.4 $47^{\prime \prime} \cdot 3$ resolution. The contours show the radio continuum emission and are similar to those in Fig.1. The frequency dependence of the primary beam was removed in a post-imaging step. 


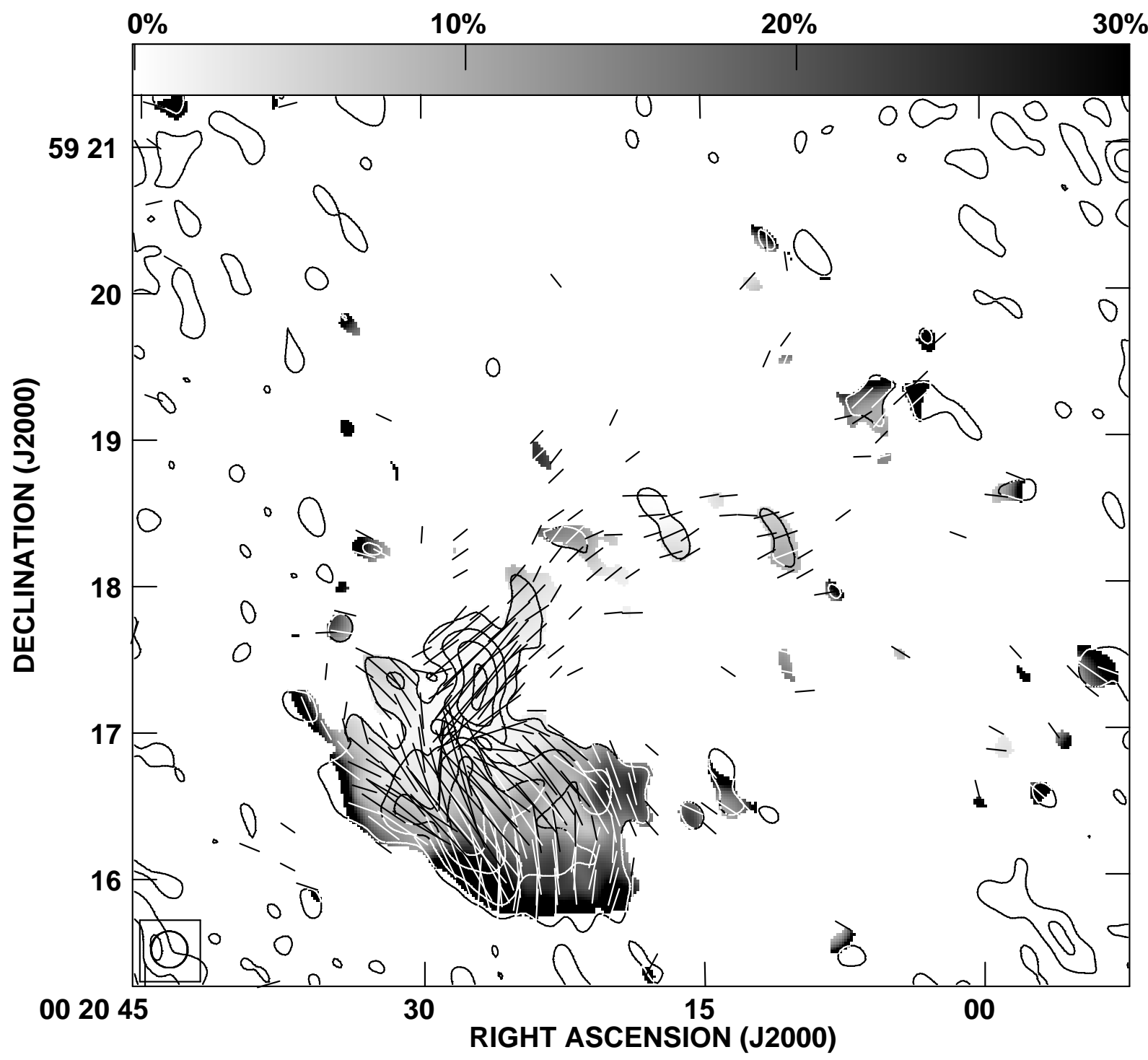

Fig. 4.- Polarized intensity and magnetic field orientation overlaid on the fractional polarization (grey scale) at 15" resolution. Polarized intensity contours are at 3, 6, 10, and 20 $\times 7 \mu \mathrm{Jy}_{\text {beam }}{ }^{-1}$. The vector length is proportional to the polarized intensity, where $10^{\prime \prime}$ is equivalent to $22 \mu \mathrm{Jy}_{\text {beam }}{ }^{-1}$ (corrected for primary beam attenuation). 


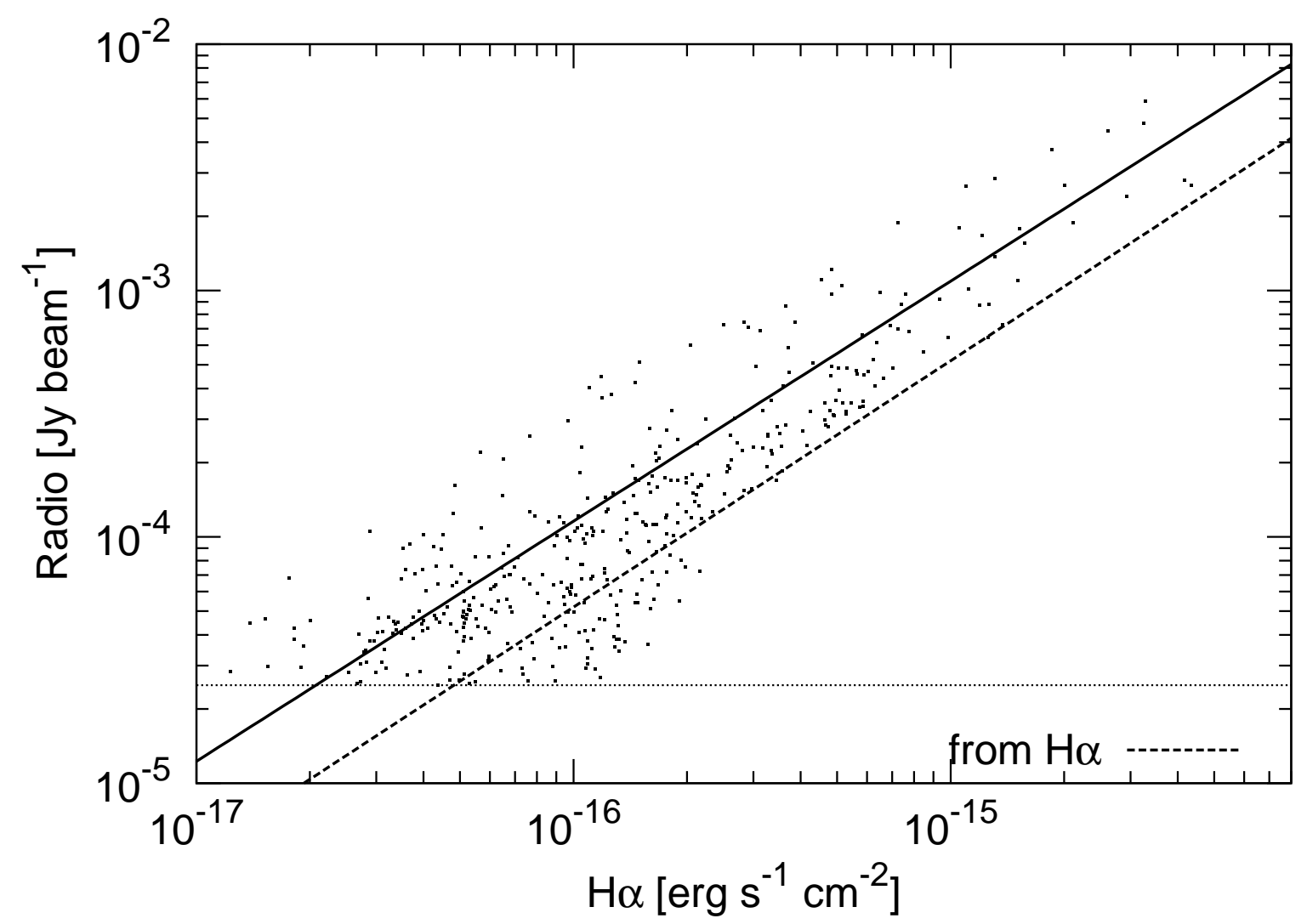

Fig. 5.- Comparison of the flux densities of the radio continuum emission at $6 \mathrm{~cm}$ with that of $\mathrm{H} \alpha$ at a scale of $50 \mathrm{pc}$. The solid line shows a fit to the data as described in the text. The dashed line shows the expected contribution based on $\mathrm{H} \alpha$ to the radio continuum via thermal free-free emission (see text for details). The horizontal line indicates $5 \times$ the $\mathrm{rms}$ noise level in the radio map, where we clipped the radio data. 